\title{
Microelastic Mapping of Living Cells: Changes in Relative Elasticity Between Nuclear and Cytoplasmic Regions of Mitotic MDCK Cells
}

\author{
Emad A-Hassan and Jan H. Hoh
}

Department of Physiology, Johns Hopkins University School of Medicine, Baltimore, MD 21205

Mechanical forces play a major role in the physiology of eukaryotic cells. Studies of the mechanical properties of cells are important for understanding their function in many physiological and pathological processes. Cytomechanics is an important factor in morphogenesis, in control of gene expression and protein synthesis and several other cellular processes especially in cellular division and proliferation. Progress has been made toward understanding the mechanics of cell division in a wide variety of organisms and cell types using several methods including molecular biology, light and electron microscopy, as well as various force transducers, methods including microneedles, laser and magnetic tweezers, and the atomic force microscope (AFM). Measurement of cellular viscoelasticity by such techniques can provide important functional information about the physiological role and mechanical properties of cellular components (in particular the cytoskeleton). Mitosis is a highly complex physiological and biochemical process. Although cellular structures and molecules involved in mitosis have been extensively characterized, the biomechanics of cell division remains poorly understood.

The AFM is emerging as a very valuable tool for studying biological samples. Besides its ability to acquire detailed surface images of various samples under physiological conditions, it also provides the means for examining sample-sample interactions and mechanical properties of samples [1]. These types of measurements are mainly performed by acquiring forces versus distant curves over the sample under consideration and then extracting elastic moduli [2]. Alternatively, we have developed an approach for relative microelastic mapping, referred to as force integration to equal limits (FIEL mapping), which is based on the fact that work done by the AFM cantilever during a force curve is related to the elasticity of the sample [3]. FIEL mapping has a number of advantages over determining local elastic moduli; there is no need to calibrate the cantilever, determine the tip radius, or know the precise tip-sample contact point in each curve.

Here we have used AFM and FIEL mapping to investigate the mechanical properties of mitotically active Madine Darby Canine Kidney (MDCK) cells. Microelastic maps were produced by collecting arrays of force curves, typically 64x64 curves, over subconfluent MDCK cells. Individual force measurements were made on a time scale, about 1 second, were viscous contributions were small. Low resolution FIEL maps of mature monolayers of MKCK cells typically appear similar to the topographic images with minor variations. Several distinct regions of the cell can be identified, predominantly the cell boundaries and the nucleus being the large structure in the center of the cell. Sub-confluent, mitotically active, MDCK cells have an elongated morphology. FIEL maps on these cells, in general, show similar features in elasticity to those obtained on mature monolayers. In the mitotically active MDCK cells, two distinct types of mechanical states can be seen (Figure 1). The first of which, described earlier, the FIEL maps show a soft intact nuclear region. In the second state, the nucleus, which appears to be divided in two parts by a soft band, is considerably stiffer than the cell body. These differences of relative elasticity appear to be related to the cell cycle, and are in agreement with point measurements made on bovine embryo skin and muscle cells [4]. 


\section{References}

[1] A. Vinckier and G. Semenza, FEBS Lett. (1998) 12.

[2] M. Radmacher et al., Biophys. J. (1996) 556.

[3] A-Hassan et al., Biophys. J. (1998) 1564.

[4] J.A. Dvorak and E. Nagao, Exp. Cell Res. (1998) 69.

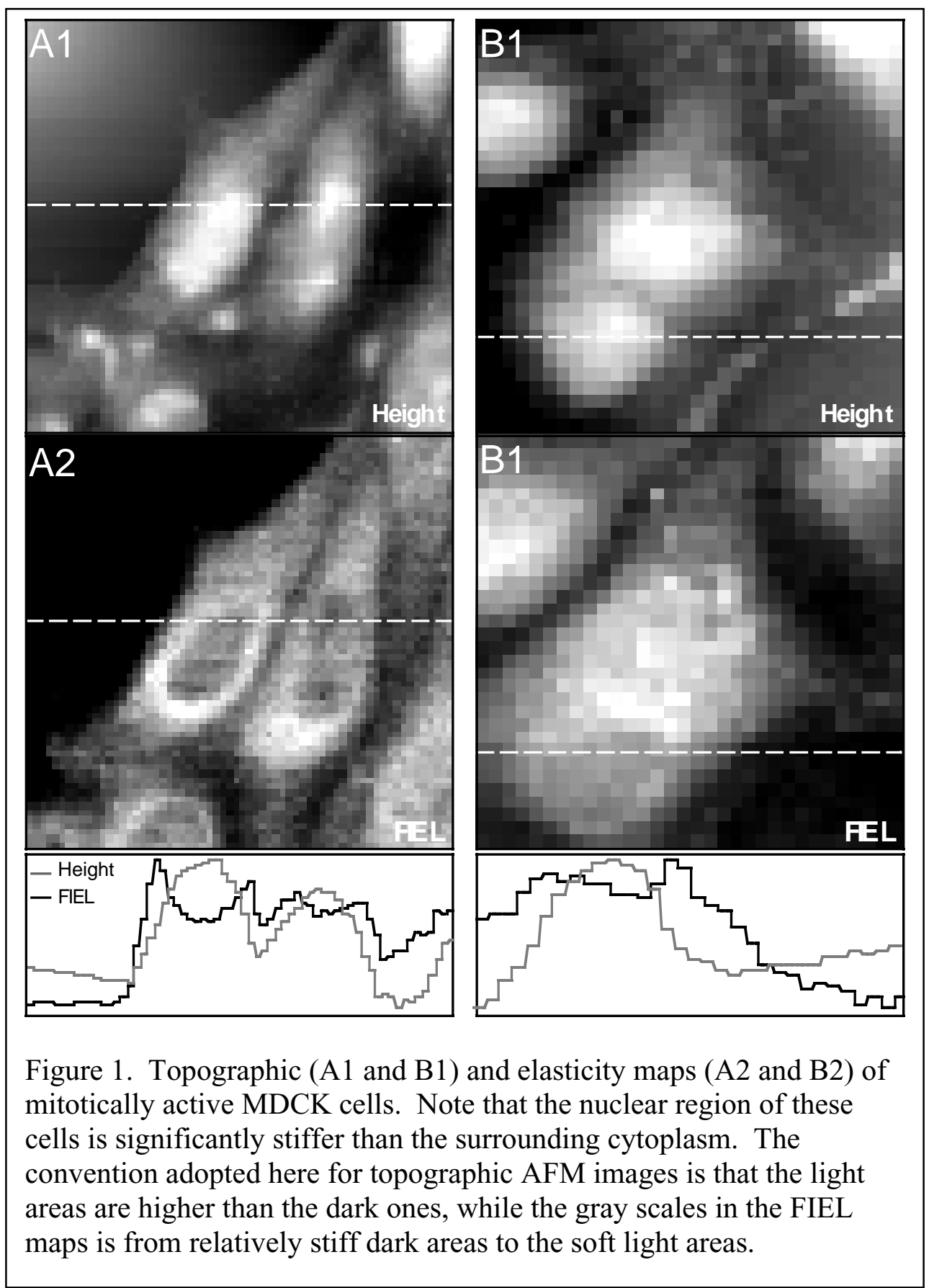

